\title{
Opioid Discontinuation Among Patients Receiving High-Dose Long-Term Opioid Therapy in the Veterans Health Administration
}

\author{
Taeko Minegishi, MS, PhD ${ }^{1,2,3}$ (D) , Melissa M. Garrido, $P h D^{1,2}$, Michael Stein, MD², \\ Elizabeth M. Oliva, $P h D^{4,5}$, and Austin B. Frakt, $P h D^{1,2,6}$
}

'Partnered Evidence-based Policy Resource Center, VA Boston Healthcare System, , MABoston, USA; ${ }^{2}$ Department of Health Law, Policy \& Management, Boston University School of Public Health, Boston, MA, USA; ${ }^{3}$ Bouvé College of Health Sciences, Northeastern University, Boston, MA, USA; “VA Office of Mental Health and Suicide Prevention, VA Program Evaluation and Resource Center, VA Palo Alto Healthcare System, Menlo Park, CA, USA; ${ }^{5}$ VA Center for Innovation to Implementation, VA Palo Alto Healthcare System, Menlo Park, CA, USA; ${ }^{6}$ Department of Health Policy and Management, Harvard T.H. Chan School of Public Health, Cambridge, MA, USA.

BACKGROUND: Prior opioid discontinuation studies have focused on one of two characteristics of opioid prescribing, its duration (long term vs not) or dosage (high vs low). Questions remain about the experience of patients with highdose, long-term opioid therapy (HLOT) prescriptions who are likely to be at the highest risk for adverse events.

OBJECTIVE: We address the following questions among the Veterans Health Administration (VHA) patients receiving HLOT: 1), How has the prevalence of discontinuation of opioids changed over time? 2), How do patient characteristics vary between those who do and do not discontinue? And 3), how does the prevalence of discontinuation vary geographically?

DESIGN: A retrospective observational study of VHA patients with HLOT between fiscal year (FY) 2014 and FY2018.

PARTICIPANTS: We identified 1,281,330 patients from VHA outpatient opioid prescription data with at least a 1-day opioid supply between FY2014 and FY2018. We identified and excluded those receiving palliative care or diagnosed with metastatic cancer.

MAIN MEASURES: For a given patient and month, a patient having a 3-month moving average of $\geq 90$ daily morphine milligram equivalent (MME) was defined as having HLOT. Similarly, we used a three-month average MME of zero as discontinuation.

KEY RESULTS:The prevalence of discontinuation among patients with HLOT increased from $6.3 \%$ in FY2014 to 7.8\% in FY2018. Across the years, patients who discontinued were younger, less likely to be married, and more likely to have comorbidities related to substance use disorders compared with patients who continued to receive HLOT. Incidence of discontinuation among those with HLOT increased in more than half (64\%) of the 129 VHA medical centers.

Prior Presentations AcademyHealth Annual Research Meeting June 24, 2019

Electronic supplementary material The online version of this article (https://doi.org/10.1007/s11606-020-06252-9) contains supplementary material, which is available to authorized users.

Received February 3, 2020

Accepted September 17, 2020

Published online November 3, 2020
CONCLUSION: Prevalence of patients receiving HLOT in the VHA decreased as the incidence of discontinuation increased. Further research is needed to understand the process by which patients are discontinued and to assess the relationship between discontinuation and health outcomes.

KEY WORDS: opioid; long-term high-dose opioid therapy; taper; discontinuation; Veterans Health Administration.

J Gen Intern Med 35(Suppl 3):S903-S9

DOI: $10.1007 / \mathrm{s} 11606-020-06252-9$

() Society of General Internal Medicine (This is a U.S. government work and not under copyright protection in the U.S.; foreign copyright protection may apply) 2020

\section{INTRODUCTION}

The Veterans Health Administration (VHA) released an Opioid Safety Initiative in 2013 to improve the safety of opioid prescribing while expanding alternative pain therapies [1]. In an effort to reduce the risks of opioid-related harms, in 2016 the Center for Disease Control and Prevention also released a guideline for prescribing opioids for chronic pain [2], which has been endorsed by medical professionals and health policy experts $[3,4]$. The guideline recommends that clinicians limit initial opioid prescription dosing for opioid-naïve patients to < 90 morphine milligram equivalent (MME) per day, a threshold based on expert consensus. However, there is growing concern that the guideline is being misapplied to inappropriately discontinue opioids for patients who have received $\geq 90$ MME/day for an extended period [3-6].

Recent studies suggest tapering long-term opioid therapy (LOT; $\geq 90$ days) patients can be successful, especially when patients consent, understand the benefits, and are closely monitored by their health care providers [7-11]. The benefits of tapering and discontinuation are reported as improvement in pain, function, and quality of life [8]. Discontinuation and tapering efforts have helped reduce the high-dose ( $\geq 90 \mathrm{MME})$ prescribing rate in the USA by $25 \%$, from 6.7 to 5.0 per 100 persons between 2015 and 2017. Concurrently, the median duration of an opioid prescription increased by $33 \%$, from 15 
to 20 days [12] and prescription duration of 30 days or longer increased by $38 \%$ (from 18 to 25 per 100 persons) between 2006 and 2017 [13]. This contrasts with experience in the VHA, where a 35\% decline in LOT between 2012 and 2016 has been observed [14]. However, it remains unclear exactly how to safely taper patients with high-dose, long-term opioid therapy (HLOT). As a result, there is concern that opioid tapering and discontinuation may lead to unmanaged pain and withdrawal symptoms, which are associated with a greater probability of adverse events, suicide attempts and death due to overdose of illicit opioids [3, 15-18].

Prior tapering and discontinuation studies have focused on either of the two greatest concerns about opioid prescribing, duration, and dose, but have rarely focused on patients with both. Questions remain about the experience of such patients, who are at the highest risk for adverse events. Our study directly addresses this gap in the literature by studying the population receiving HLOT. Among patients with an opioid prescription from the VHA between 2013 and 2018 who received high-dose $(\geq 90$ MMEs per day) and long-term $(\geq$ 90 days) opioid therapy, we address the following questions: 1), How has the rate of discontinuation in this population changed over time? 2), How do patient characteristics vary between those who do and do not discontinue? And 3), how does prevalence of opioid discontinuation vary geographically across VHA medical centers?

\section{METHODS}

Cohort. We identified 1,281,330 patients from all 129 VHA medical centers throughout the USA. We included VHA outpatient opioid prescription data with at least a 1-day supply of opioids for pain between fiscal years (FYs) 2014 (October 1, 2013-September 30, 2014) and FY2018 (October 1, 2017 September 30, 2018). We identified and excluded patients receiving palliative care or those diagnosed with metastatic cancer (ICD-9 and 10 codes in Appendix Table 1) [19, 20] during the study period for whom high-dose or long-term opioid therapy may be appropriate [21]. After exclusions, we examined dose and duration of opioid prescriptions among a cohort of 1,123,247 patients. Patient sociodemographic and clinical characteristics, including age, gender, race, marital status, Elixhauser comorbidities [19, 22], opioid use disorder (OUD), and number of months on opioid prescriptions, and all-cause mortality were collected from VHA administrative databases. The study protocol was approved by the VA Boston Institutional Review Board (IRB 3069; R\&D\#3244-X).

Opioid use. To standardize the dosages across prescriptions with different formulations, quantities, and days of supply, all opioid prescriptions were converted using CDC's morphine equivalent conversion factors [23]. All opioid prescriptions from the same month were added together to calculate the
MME per day in a given month for each patient. (See Appendix Table 2 for list of opioid prescription names.) From these data, we calculated the 3-month moving average of daily MME to avoid non-meaningful idiosyncrasies of briefly overlapping prescriptions and small gaps between prescription fills. The 3 months included the given month and the two prior months (e.g., the value for March included data from January, February, and March) and hereinafter, average MME refers to a three-month moving average daily MME unless otherwise specified. All analyses were performed at the patient-month level.

States of Opioid Use. Our primary focus was patients with HLOT who subsequently discontinued. HLOT was defined as an average of $\geq 90$ MME. Similarly, we defined discontinuation as an average MME of zero, indicating no opioid prescriptions for three consecutive months. Patients with an average MME $>0$ and $<90$ were classified as not receiving HLOT (non-HLOT). Each month, patients were categorized into one of four states based on the average MME: HLOT, opioid discontinuation, non-HLOT, or death. Death was defined as all-cause death, with dates based on death records for all veterans.

\section{Analysis}

To assess the prevalence of transitions from HLOT to other states, we used a Markov chain model for each year. These Markov models captured transitions between states from the beginning to the end of a FY but ignored within-year transitions. For example, a patient with HLOT in the first month of the FY (October) could end up in one of 4 states by the end of the year (September of the following calendar year): stay in the same state (HLOT), or transition into non-HLOT, discontinuation, or death. Patients were included in the analysis across multiple years until death (death is a terminal state).

Our first objective was to identify time trends of transition to discontinuation among patients receiving HLOT. The incidence of discontinuation for this group was calculated using the number of patients in the HLOT state at the beginning of the FY as the denominator and number of patients who transitioned from HLOT to discontinuation by the end of the FY as the numerator. We also assessed trends in the rates of the transition of patients in HLOT to death or Non-HLOT states.

For a single FY (2016, the midpoint of our study window), we assessed differences in patient characteristics between patients in HLOT who transitioned to discontinuation and patients who remained in the HLOT state. Baseline characteristics were calculated for the period 1 year prior to each FY and compared using standardized differences where the comparison of two groups can be assessed independent of sample size. We denoted the differences between the two groups as meaningful if the standardized difference was $>10 \%$ [24]. 
In addition, at the medical center level, we analyzed the geographic variation of the rate of discontinuation among patients receiving HLOT. Most patients $(>85 \%)$ received their opioid prescriptions from a single medical center; when a patient received prescriptions from multiple medical centers the patient was assigned to the medical center where the plurality of prescriptions was received. Facility mental health (MH) capacity, number of $\mathrm{MH}$ staff per 10,000 unique patients, was also collected to characterize one potentially important factor in the differences between medical centers with very large increases or decreases in discontinuation rates over time.

Finally, we conducted the following sensitivity analyses and robustness checks. First, because we can only characterize VHA opioid prescribing, to address concerns that patients receiving HLOT who apparently discontinued may have ended VHA care, we excluded patients who did not have at least one outpatient appointment after 6 months of their last prescription month. Second, we examined whether providers were more likely to discontinue opioids among patients receiving HLOT with an OUD diagnosis in the baseline period, who generally should not be receiving HLOT. To do this, we stratified the patients with HLOT by OUD diagnosis and explored the difference in discontinuation rates.

\section{RESULTS}

Cohort Characteristics. Of the 1,123,247 patients in our cohort, the average age is 59.6 years old, over $90 \%$ are male, and more than $70 \%$ are white (Table 1). Less than $6 \%$ are Hispanic or Latino, more than half are married, and $14 \%$ of the cohort died during the study period. The average MME for each patient during the entire study period is 15.2 with a standard deviation of $33 ; 7.7 \%$ appear in the HLOT state at least once, and $78 \%$ of patients with HLOT discontinue at least once during the study period.

Table 1 Patient Characteristics of the Study Cohort $(N=1,123,247)$

\begin{tabular}{ll}
\hline \hline Patient characteristics & $\begin{array}{l}\text { Mean [SD] or } \\
\%\end{array}$ \\
\hline Age & $59.6[13.8]$ \\
Male, \% & 91.4 \\
Race: White, \% & 73.7 \\
Race: Black, \% & 18.4 \\
Race: Other, \% & 7.4 \\
Hispanic, \% & 5.9 \\
Married, \% & 57.9 \\
Deceased during the study, \% & 13.9 \\
Opioid prescription pattern: & $15.2[33.3]$ \\
Average MME for each patient during the entire & \\
study period & 7.7 \\
HLOT state for at least 1 month during the study, \% & 78 \\
Discontinued at least 1 month during the study, \% &
\end{tabular}

Temporal Analysis. At the start of our analysis period, FY2014, 49,708 patients $(4.4 \%)$ were in the HLOT state, $416,047(37 \%)$ in the discontinued state, and 657,492 (59\%) in the non-HLOT state. Figure 1 characterizes the output of the Markov chain model for FY2014. Among the 49,708 patients receiving HLOT at the start of FY2014, 35,921 (72\%) remained in continued to receive HLOT at the end of FY2014; $3128(6.3 \%)$ patients transitioned to discontinuation; 8935 (18\%) patients transitioned to non-HLOT; and 1724 (3.5\%) patients died.

A sensitivity analysis that stratified patients receiving HLOT with and without an OUD diagnosis during the baseline year (HLOT + OUD) showed that the former group is more likely to discontinue. Over time, on average, $11 \%$ of patients with HLOT+OUD discontinued by the end of each FY, whereas only $7 \%$ of patients with HLOT without an OUD diagnosis discontinued.

A separate Markov chain model was calculated for each year in the study period to assess the annual trends of transitions among patients receiving HLOT state transitions (Table 2). Prevalence of patients receiving HLOT at the start of the FY decreased between FY2014 and FY2018, from $74.4 \%$ (49,708 patients with HLOT among 707,200 patients prescribed opioids in FY2014) to $49.6 \%$ (22,999 patients with HLOT among 463,779 patients prescribed opioids in FY2018). Incidence of opioid discontinuation among patients with HLOT increased, from $6.3 \%$ in FY2014 to $7.8 \%$ in FY2018. The incidence of all-cause death was relatively stable over the same time period, ranging between 3.1 and $3.5 \%$. Between FY2014 and FY2018, the proportion of patients who started the FY as receiving HLOT remained high, over $60 \%$, though it decreased from 72

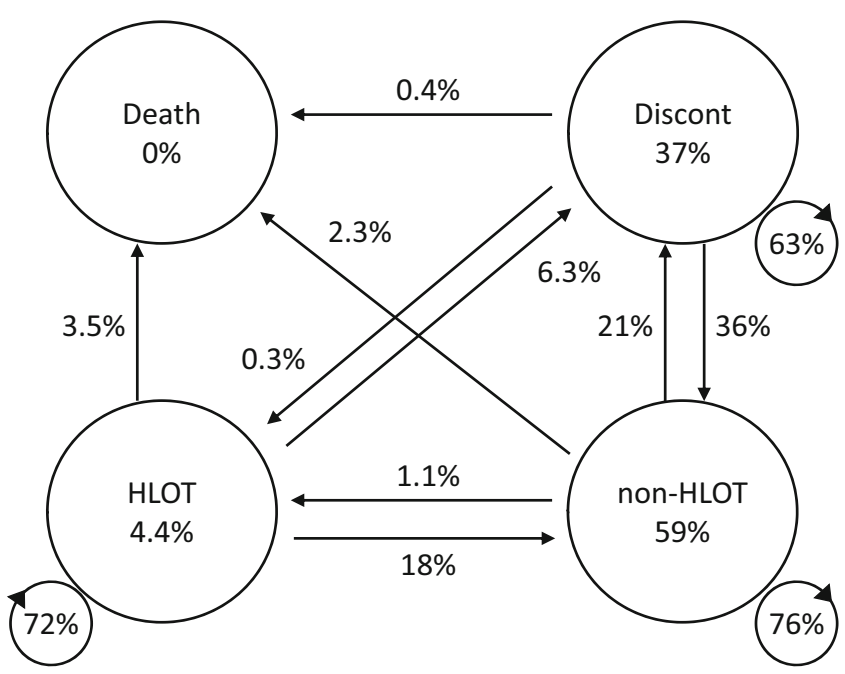

Figure 1 Markov chain models for fiscal year 2014. Percentages may not total 100 due to rounding. All 1,123,247 patients receiving opioids were classified into one of four states; high-dose, long-term opioid therapy (HLOT; $n=49,708)$, non-HLOT $(n=657,492)$, discontinued (Discont; $n=416,047$ ), or death. The numbers inside the large circles are the prevalence at the start of the year. The numbers next to the arrows are transition probabilities. 
Table 2 Transition Probabilities for Patients Receiving High-dose, Long-term Opioid Therapy (HLOT) at the Start of Each Fiscal Year Between 2014 and 2018

\begin{tabular}{llllll}
\hline \hline FY & HLOT & $\begin{array}{l}\text { Non- } \\
\text { HLOT }\end{array}$ & Discontinued & Death & Total \\
\hline 2014 & 35,921 & 8935 & $3128(6.3 \%)$ & 1724 & 49,708 \\
& $(72 \%)$ & $(18 \%)$ & $2.5 \%)$ & \\
2015 & 31,479 & 7967 & $2992(6.8 \%)$ & 1484 & 43,922 \\
& $(72 \%)$ & $(18 \%)$ & & $(3.4 \%)$ & \\
2016 & 26,604 & 7693 & $2786(7.3 \%)$ & 1225 & 38,308 \\
& $(69 \%)$ & $(20 \%)$ & & $(3.2 \%)$ & \\
2017 & 20,051 & 7638 & $2460(7.9 \%)$ & 1026 & 31,175 \\
& $(64 \%)$ & $(25 \%)$ & & $(3.3 \%)$ & \\
2018 & 14,732 & 5747 & $1798(7.8 \%)$ & 722 & 22,999 \\
& $(64 \%)$ & $(25 \%)$ & & $(3.1 \%)$ & \\
\hline
\end{tabular}

Markov chain models were calculated for fiscal years (FY) 2014 through 2018. The proportion of patients who received high-dose, longterm opioid therapy (HLOT) at the beginning of the FY and stayed in HLOT, transitioned to non-HLOT, discontinued, or died were calculated for each $F Y$ and presented in the table above

to $64 \%$ between FY2014 and FY2018. This coincided with an increase in the proportion of patients who transitioned from HLOT to Non-HLOT (from 18 to $25 \%$ ) or discontinued (from 6.3 to $7.8 \%$ ) over the same years. (The Markov chain transition models for FY2014 through FY2018 can be found in Appendix Fig. 1)

Patient Characteristics. For FY2016, we compared the baseline characteristics of patients receiving HLOT who transitioned to the discontinued state and those who continued with HLOT. Table 3 shows the results: 26,604 patients remained in the HLOT state and 2786 patients transitioned to discontinuation. On average, patients who transitioned to discontinuation were younger, less likely to be married, and more likely to have an alcohol, opioid, or other drug use disorder, compared with patients who continued to receive HLOT. Also, during the baseline year, compared with patients who stayed in HLOT, patients who transitioned to discontinued had a lower average opioid MME (187.7 MME vs. 170.0 MME) and fewer number of months in HLOT state (10.8 vs. 9.8 months). The baseline characteristic of patients receiving HLOT who transitioned to any state other than HLOT state was no different than patients who stayed in HLOT, patients who died were older and sicker compared with patients who stayed in HLOT (results shown in Appendix Table 3). We ran the comparisons for each year and found consistent results (results available upon request).

Geographic Variation. Across the 129 VHA medical centers, the transition probabilities for patients who were receiving HLOT at the start of the FY and discontinued at the end of the FY ranged between 1.5 and $15.2 \%$ in FY2014 and between 0 and $25.0 \%$ in FY2018. Between FY2014 and FY2018, rates of discontinuation increased at eighty-two medical centers $(64 \%)$ and decreased at forty-seven medical centers $(36 \%)$. The changes in probability of discontinuation between FY2014 and FY2018 at each medical center ranged between -10.2 and $17.9 \%$. The medical centers with large positive or negative changes in discontinuation rate between FY2014 and FY2018 (defined as those at or above the 75th percentile of absolute difference, or 5.9\%) are shown in Figure 2. Between FY2014 and FY2018, seven medical centers experienced large drops (red triangles) and twenty-four medical centers experienced large increases (blue squares) in discontinuation rate. Large decreases occurred in the Northeast and Upper Midwest regions. The medical centers with large increases in discontinuation were scattered throughout the country. In FY2014, MH staffing ratios were higher among the medical centers with large decreases in discontinuation compared with medical centers with large increases (26.9 vs 21.0 staff per 10,000 unique patients, $24 \%$ standardized difference). We also found that medical centers with large decreases in discontinuation had, on average, a lower proportion of patients with HLOT per total unique patients $(0.64 \%$ vs $0.86 \%$ ), and fewer patients receiving HLOT, on average, compared with medical centers with large increases (209 vs 350 patients in HLOT).

\section{DISCUSSION}

We analyzed VHA outpatient opioid prescriptions data between FY2014 and FY2018 and identified, by year, patients with HLOT. Between FY2014 and FY2018, the number of patients receiving HLOT decreased from 49,708 to 22,999 patients (53.7\% decrease in overall patients with HLOT). Among patients with HLOT at the start of the FY, the proportion of discontinued at the end of the FY increased during these years from 6.3 to $7.8 \%$ in FY2018. Our findings are consistent with the trend reports from VHA and others that high-dose prescribing rate and average dose level of persons receiving opioids declined in recent years $[1,12,13]$, though the main population we examined were those receiving HLOT at the start of each FY. These findings may be due to several VHA policies implemented to improve opioid safety (more details on these policies can be found elsewhere) $[1,25]$.

Hadlandsmyth and colleagues [14] reported a substantial decline in initiation of LOT between 2015 and 2016. Of existing long-term opioid users in their study (which included both HLOT and non-HLOT), $21.8 \%$ either switched from long-term to short-term (less than 90 days) opioid therapy or discontinued. These numbers are similar to our finding that among the patients receiving HLOT in FY2016, 20\% transitioned into non-HLOT and $7.3 \%$ discontinued. However, Hadlandsmyth and colleagues [14] focused on patients with LOT of any dose level and only examined transitions in prescription duration within a year. Our analysis examines patients who receive HLOT and explicitly includes transitions to lower dose, discontinuation, or death. 
Table 3 Standardized Differences of Characteristics of Patients with High-dose, Long-term Opioid Therapy (HLOT) between patients who stayed in HLOT and transitioned to discontinuation in fiscal year 2016

\begin{tabular}{|c|c|c|c|c|}
\hline Variable groups & Variables & $\begin{array}{l}\text { Stayed in } \\
\text { HLOT }\end{array}$ & $\begin{array}{l}\text { Transitioned to } \\
\text { discontinuation }\end{array}$ & $\begin{array}{l}\text { Standardized } \\
\text { difference }(\%)\end{array}$ \\
\hline Number of patients in each group & & 26,604 & 2786 & - \\
\hline \multirow[t]{8}{*}{ Patient characteristics } & Age & 60.3 & 57.8 & 22.1 \\
\hline & $\begin{array}{l}\text { Number of months in HLOT state during the } \\
\text { baseline year }\end{array}$ & 10.8 & 9.8 & 32.7 \\
\hline & Average MME during the baseline year & 187.7 & 170.0 & 14.4 \\
\hline & Male & $93.5 \%$ & $93.8 \%$ & -1.3 \\
\hline & Married & $58.1 \%$ & $51.3 \%$ & 13.7 \\
\hline & Race; White & $83.1 \%$ & $82.9 \%$ & 0.5 \\
\hline & Race: Black & $9.4 \%$ & $9.7 \%$ & -1.0 \\
\hline & Race: Other & $7.5 \%$ & $7.4 \%$ & 0.4 \\
\hline \multirow{8}{*}{$\begin{array}{l}\text { Elixhauser comorbidities* plus } \\
\text { opioid use disorder }\end{array}$} & Alcohol abuse & $7.1 \%$ & $11.5 \%$ & -14.8 \\
\hline & Depression & $53.9 \%$ & $58.4 \%$ & -9.2 \\
\hline & Diabetes - uncomplicated & $30.2 \%$ & $26.5 \%$ & 8.4 \\
\hline & Drug abuse & $13.1 \%$ & $22.2 \%$ & -24.1 \\
\hline & Hypertension-uncomplicated & $58.8 \%$ & $54.9 \%$ & 7.8 \\
\hline & Other neurological disorder & $20.7 \%$ & $18.6 \%$ & 5.1 \\
\hline & Opioid use disorder ${ }^{\dagger}$ & $7.9 \%$ & $\begin{array}{l}10.0 \% \\
13.7 \%\end{array}$ & $\begin{array}{l}5.1 \\
-18.9\end{array}$ \\
\hline & Paralysis & $3.6 \%$ & $2.7 \%$ & 5.1 \\
\hline
\end{tabular}

MME, morphine milligram equivalent per day; HLOT, high-dose, long-term opioid therapy

*This table only presents the Elixhauser comorbidities that had standardized difference greater than 5\%. The full list is available in Appendix Table 3 †Opioid use disorder is counted towards drug abuse using Elixhauser comorbidity definitions. Drug abuse includes opioids, cocaine, hallucinogen, sedative, cannabis, and other drugs

We also compared characteristics of patients receiving HLOT who remained in that state to those who transitioned to the discontinued state. Across the years, patients who discontinued were younger, less likely to be married, and had more comorbidities related to substance use disorders compared with patients who stayed on HLOT. Patients with HLOT + OUD is more likely to be discontinued compared with patients without OUD (11\% vs $7 \%)$, perhaps due to a higher risk of serious adverse events; health care providers are encouraged to taper or discontinue opioid prescriptions from such at-risk patients or change them to less dangerous opioid agonist therapies such as buprenorphine [26, 27]. Lovejoy and colleagues [28] reported that aberrant behaviors were among the most commonly reported reason for clinicians to discontinue LOT among patients with substance use disorders.

In our analysis of geographic variation, we found that incidence of discontinuation among the patients receiving HLOT increased in more than half $(64 \%)$ of the 129 facilities throughout the USA during this period. The higher MH staffto-patient ratio in medical centers with large decreases in

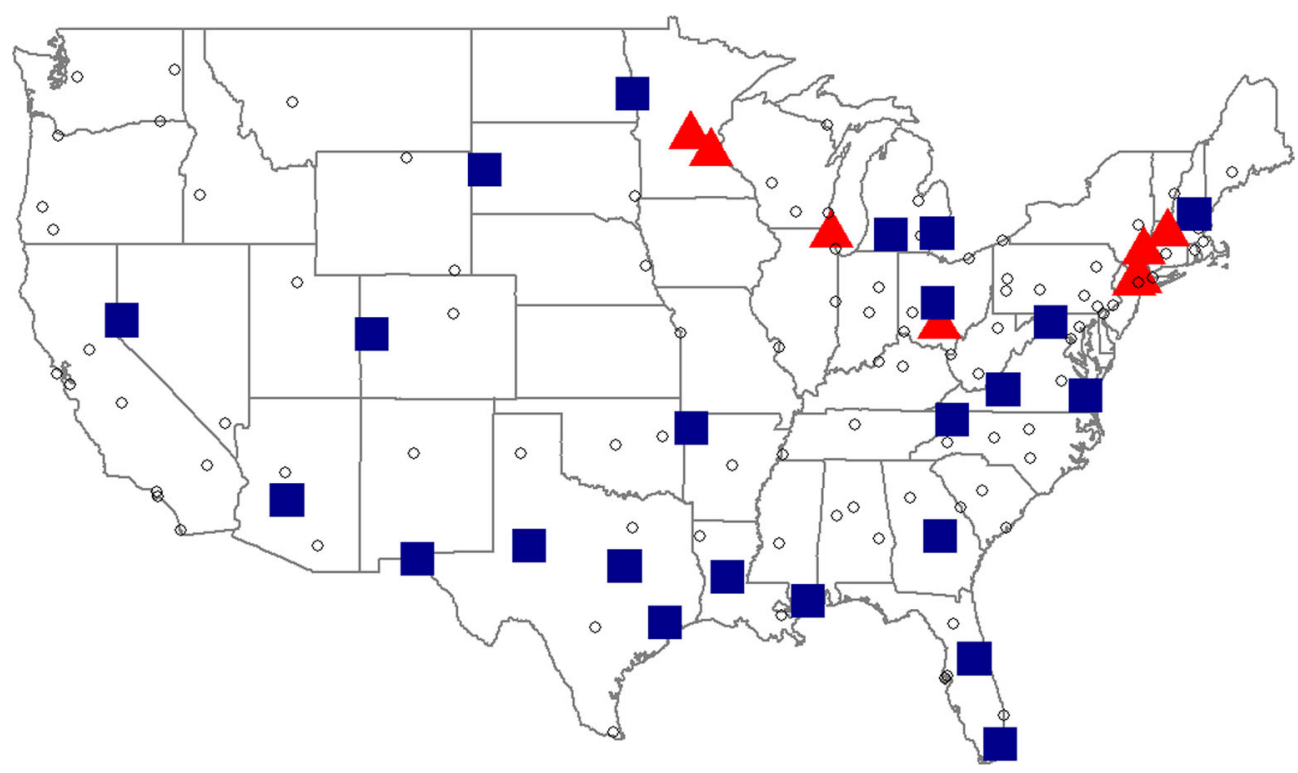

Figure 2 A US map with 126 VHA medical centers. Each point (triangle, square, or circle) represents a VHA medical center. The red triangles represent the 7 medical centers with large decreases in end of the fiscal year discontinuation rate among patients on high-dose, long-term opioid therapy (HLOT) at the beginning of the fiscal year between fiscal years 2014 and 2018 . The blue squares represent the 24 medical centers with large increases in discontinuation rate. The open circles represent the medical centers that did not have a large increase or decrease in discontinuation rate (the absolute change in discontinuation rate between fiscal year 2014 and 2018 was less than $5.9 \%$ ). 
discontinuation rates between FY2014 and FY2018 may have contributed in two ways. First, higher capacity may reflect more resources which may allow providers to manage patients' pain without escalating to HLOT (on average, 209 patients receiving HLOT for medical centers with large decreases vs 350 patients receiving HLOT for medical centers with large increases, in FY2014). Second, HLOT requires regular checkups and laborious follow-up; thus, medical centers with lower capacity may be more likely to quickly discontinue opioid prescriptions to reduce treatment burden. Under optimal conditions (i.e., with patients' consent and patientcentered tapering), increases in discontinuation rates would be a positive sign for risk reduction of this population. However, multiple factors could contribute to changes in discontinuation rate and outcomes over time.

There are several limitations to this study. First, though we could account for all VHA outpatient opioid prescriptions, we did not capture any opioid prescriptions from non-VHA prescribers. Therefore, we may be underestimating the prevalence of HLOT (or non-HLOT) among patients and over-estimating the incidence of discontinued patients. Work by Chui and colleagues [29] showed that Veterans with dual use of VHA and Medicare were significantly more likely to be receiving LOT compared with Veterans with only VHA or Medicare. From our sensitivity analysis, less than $20 \%$ of patients with HLOT who discontinued did not have an outpatient VHA appointment 6 months after their opioid prescription expired, suggesting that they left VHA care. When excluding these patients from our analysis, the incidence of discontinuation among patients with HLOT decreased by approximately 1 percentage point. The annual trend is consistent with the main analysis and found an increase in discontinuation rate between FY2014 and FY2018 (5.1\% and 6.7\%, respectively). Second, our annual Markov chain analysis relies on the first and last months of years to determine initial and final states and transitions between them. Thus, it does not account for changes in patients' status within a year. Finally, as this is an observational study with VHA patient data, we do not interpret reported associations causally.

\section{CONCLUSION}

We examined temporal changes and geographic variation in opioid prescribing for patients receiving HLOT in the VHA, an understudied cohort with high risk of serious adverse events. Between FY2014 and FY2018, the prevalence of patients with HLOT decreased as the incidence of discontinuation increased. The mechanisms by which these patients discontinued are not well understood and may depend on both patient and provider factors, giving rise to the observed geographical variation. Patients, providers, and policy-makers should be cautious in how they engage in and encourage opioid tapering and discontinuation, as rapid withdrawal of prescription opioids may cause more harm than good for some patients. Furthermore, more research and development of evidence-based policy is needed to guide clinicians in how best to support patients who have recently discontinued their HLOT.

Acknowledgments: We thank Dr. Jodie Trafton (VH Office of Mental Health and Suicide Prevention) for the insightful discussions.

Corresponding Author: Taeko Minegishi, MS, PhD; Partnered Evidence-based Policy Resource Center, VA Boston Healthcare System, MA, Boston, USA (e-mail: taeko.minegishi@va.gov).

Funding This work is supported by the Department of Veterans Affairs, Veterans Health Administration, Office of Research and Development (HSR\&D SDR 16-196; QUERI PEC 16-001).

\section{Compliance with Ethical Standards:}

The study protocol was approved by the VA Boston Institutional Review Board (IRB 3069; R\&D\#3244-X).

Conflict of Interest: The authors declare that they do not have a conflict of interest.

Disclaimer: The contents do not represent the views of the U.S. Department of Veterans Affairs, the United States Government, Northeastern University, Boston University, or Harvard University.

\section{REFERENCES}

1. Gellad WF, Good CB, Shulkin DJ. Addressing the Opioid Epidemic in the United States: Lessons From the Department of Veterans Affairs. JAMA Internal Medicine. 2017;177(5):611-612.

2. Dowell D, Haegerich T, Chou R. CDC Guideline for Prescribing Opioids for Chronic Pain - United States, 2016. Centers for Disease Control and Prevention; 2016:1-50.

3. Rubin R. Limits on Opioid Prescribing Leave Patients with Chronic Pain Vulnerable. JAMA. 2019;321(21):2059-2062.

4. Dowell D, Haegerich T, Chou R. No Shortcuts to Safer Opioid Prescribing. $N$ Engl J Med. 2019;380(24):2285-2287.

5. Kroenke K, Alford DP, Argoff C, et al. Challenges with Implementing the Centers for Disease Control and Prevention Opioid Guideline: A Consensus Panel Report. Pain Medicine. 2019;20(4):724-735.

6. Kertesz SG, Gordon AJ. A crisis of opioids and the limits of prescription control: United States: Opioids and the limits of prescription control. Addiction. 2019;114(1): 169-180.

7. Cunningham JL, Evans MM, King SM, Gehin JM, Loukianova LL. Opioid Tapering in Fibromyalgia Patients: Experience from an Interdisciplinary Pain Rehabilitation Program. Pain Med. 2016;17(9):1676-1685.

8. Frank JW, Lovejoy TI, Becker WC, et al. Patient Outcomes in Dose Reduction or Discontinuation of Long-Term Opioid Therapy: A Systematic Review. Annals of Internal Medicine. 2017;167(3):181-191.

9. Harden P, Ahmed S, Ang K, Wiedemer N. Clinical Implications of Tapering Chronic Opioids in a Veteran Population. Pain Med. 2015;16(10):19751981.

10. McPherson S, Lederhos Smith C, Dobscha SK, et al. Changes in pain intensity after discontinuation of long-term opioid therapy for chronic noncancer pain: PAIN. 2018;159(10):2097-2104.

11. Hundley L, Spardley S, Donelenko S. Assessment of outcomes following high-dose opioid tapering in a veterans healthcare system. Journal of Opioid Management. 2018;14(2):89-101.

12. Guy, Gery P., Zhang, Kun, Schieber, Lyna Z., Young, Rndall, Dowell, Deborah. County-Level Opioid Prescribing in the United States, 2015 and 2017. JAMA Internal Medicine. 2019;179(4):574-576.

13. Schieber LZ, Guy GP, Seth P, et al. Trends and Patterns of Geographic Variation in Opioid Prescribing Practices by State, United States, 20062017. JAMA Network Open. 2019;2(3):e190665. 
14. Hadlandsmyth K, Mosher H, Vander Weg MW, Lund BC. Decline in Prescription Opioids Attributable to Decreases in Long-Term Use: A Retrospective Study in the Veterans Health Administration 2010-2016. Journal of General Internal Medicine. 2018;33(6):818-824.

15. Demidenko MI, Dobscha SK, Morasco BJ, Meath THA, Ilgen MA, Lovejoy TI. Suicidal ideation and suicidal self-directed violence following clinicianinitiated prescription opioid discontinuation among long-term opioid users. General Hospital Psychiatry. 2017;47:29-35.

16. Mark TL, Parish W. Opioid medication discontinuation and risk of adverse opioid-related health care events. Journal of Substance Abuse Treatment. 2019;103:58-63.

17. Oliva EM, Bowe T, Manhapra A, et al. Associations between stopping prescriptions for opioids, length of opioid treatment, and overdose or suicide deaths in US veterans: observational evaluation. BMJ. Published online March 4, 2020:m283.

18. Rosenberg JM, Bilka BM, Wilson SM, Spevak C. Opioid Therapy for Chronic Pain: Overview of the 2017 US Department of Veterans Affairs and US Department of Defense Clinical Practice Guideline. Pain Medicine. 2018;19(5):928-941.

19. Quan H, Sundararajan V, Halfon P, et al. Coding Algorithms for Defining Comorbidities in ICD-9-CM and ICD-10 Administrative Data: Medical Care. 2005;43(11): 1130-1139.

20. Mor V, Joyce NR, Coté DL, et al. The rise of concurrent care for veterans with advanced cancer at the end of life: EOL Care for Veterans With Advanced CA. Cancer. 2016;122(5):782-790.

21. Cleary JF. Cancer Pain Management. 2000;7:12.

22. Li P, Kim MM, Doshi JA. Comparison of the performance of the CMS Hierarchical Condition Category (CMS-HCC) risk adjuster with the Charlson and Elixhauser comorbidity measures in predicting mortality. BMC health services research. 2010;10(1):245.
23. Center for Disease Control and Prevention. Opioid Morphine Equivalent Conversion Factors. Published online May 2014. Accessed June 10, 2020. https://www.cms.gov/Medicare/Prescription-Drug-Coverage/PrescriptionDrugCovContra/Downloads/Opioid-Morphine-EQ-ConversionFactors-March-2015.pdf

24. Austin PC. Using the Standardized Difference to Compare the Prevalence of a Binary Variable Between Two Groups in Observational Research. Communications in Statistics - Simulation and Computation. 2009;38(6): 1228-1234.

25. Minegishi T, Frakt A. Reducing Long-term Opioid Use in the Veterans Health Administration. Journal of General Internal Medicine. Published online February 15, 2018.

26. Edlund MJ, Steffick D, Hudson T, Harris KM, Sullivan M. Risk factors for clinically recognized opioid abuse and dependence among veterans using opioids for chronic non-cancer pain: Pain. 2007;129(3):355-362.

27. Edlund MJ, Martin BC, Devries A, Fan M-Y, Brennan Braden J, Sullivan MD. Trends in Use of Opioids for Chronic Noncancer Pain Among Individuals With Mental Health and Substance Use Disorders: The TROUP Study: The Clinical Journal of Pain. 2010;26(1):1-8.

28. Lovejoy TI, Morasco BJ, Demidenko MI, Meath THA, Frank JW, Dobscha SK. Reasons for discontinuation of long-term opioid therapy in patients with and without substance use disorders: PAIN. 2017;158(3):526-534.

29. Chui PW, Bastian LA, DeRycke E, Brandt CA, Becker WC, Goulet JL. Dual Use of Department of Veterans Affairs and Medicare Benefits on High-Risk Opioid Prescriptions in Veterans Aged 65 Years and Older: Insights from the VA Musculoskeletal Disorders Cohort. Health Serv Res. 2018;53:5402-5418.

Publisher's Note: Springer Nature remains neutral with regard to jurisdictional claims in published maps and institutional affiliations. 